\title{
A Call to Arms
}

\author{
John E. R. Staddon \\ Duke University
}

There is little doubt that behavior analysis as a field is somewhat beleaguered-at least in our elite universities, where a shrinking number of new positions are targeted for this area. Part of the problem is the decline in animal research, at least animal research of the purely behavioral variety. But the main difficulty is the assumption by most critics that behaviorism is simply a dead issue. A recent review by Jerry Fodor of a book called Thinking Without Words by José Luis Bermúdez (2003) is an example of what most cognitive psychologists think, although few are as forceful as he in expressing it. Fodor is a well-known, respected, and highly articulate philosopher of mind, a vigorous proponent of the modular view of mind, and a typical advocate of mainstream cognitive science. His piece shows behaviorists what they must deal with if their approach to science is not to go the way of ... what? Not of phlogiston theory-which was after all wrong and should have faded from view-but of Mendel, whose ideas were correct but whose work was ignored for 30 years until it was rediscovered.

Fodor's view of behaviorism is rather like most people's view of the flatearth society: Behaviorism is so obviously wrong that repeating the arguments against it is supererogatory. It is worth mentioning only to be dismissed as a sort of throat-clearing exercise before we get on to the real stuff. (No one mentions flat earthers any more, but sneering at behaviorism is still de rigueur among cognitivists. They doth protest too much, methinks ...)

Address correspondence to the author at the Department of Psychology: Experimental, Duke University, Box 90086, Durham, North Carolina 27708.
Here is the beginning of Fodor's review:

“Dr Livingstone, I presume?" Stanley was spot on: it was Dr Livingstone. Elsewise his presuming so wouldn't have become the stuff of legend. ... How did he manage to presume so cleverly? ... This seems safe: Stanley must have done some thinking. He must have inferred, on the basis of his beliefs, memories, hunches (etc.) about the situation in which he found himself, that it was Livingstone he ought to presume. "The situation in which he found himself" thus included not only whatever was perceptually available at the scene, but also a lot of cognitive commitments that Stanley brought with him. If he inferred that it was Livingstone, it must have been from those sorts of premise that he did so; he had nothing else to go on. I suppose that's all pretty much truistic. (p. 16)

So, to Fodor and his readers, it is obvious ("truistic") that explaining behavior like Livingstone's-guessing the identity of a new acquaintancerequires us to make reference to "beliefs," "memories," "hunches," and the like; "cognitive commitments" in his terms. Fodor's assertion would raise no eyebrows either among most cognitive scientists or among a random selection from the audience of the Jerry Springer show. In short, Fodor's philosophy of mind differs in no way from folk psychology. Moreover, he assumes not just that behavior is best explained in mentalistic terms, but that it must be explained in this way:

Notice, to begin with, the intimate relation between thinking and inferring. At the crucial point, Stanley's thinking must have consisted of drawing inferences from what he independently believed. It's plausible that at least some kinds of thinking just are processes of drawing inferences. It's the same for a lot of other things the mind does, such as learning, perceiving and planning. The picture that emerges is of the mind (or the brain if you prefer) as some kind of inferring machine; perhaps some kind of computing machine, since computations are themselves plausibly construed as chains of inference. 
Second, if the mind is in the inference-drawing line of work, there must be symbols in which it formulates its premises and conclusions; there are no inferences without a medium (or media) in which to couch them. That matters because you can't say just anything you like in whatever kind of symbols you choose. Pictures can't express negative or contingent propositions-it's not raining, or if it's raining that will spoil the picnic. (p. 16)

So, we must accept not just beliefs and memories but also symbols. Fodor's folk psychology apparently leads straight from inferences to Simon's (1957) view of the mind as a symbolmanipulating device.

Fodor's psychology assumes that there are things going on in the head that cannot be directly observed. What are these things? If you are a neuroscientist, they are neural events. If you are Fodor, they are the mental ingredients of folk psychology- "vehicles of thought ... not very different from ... 'natural languages' (English, German, whatever)" (p. 16). But, to Fodor's distress, in the history of our field behaviorism keeps breaking out:

Over the years, psychologists have worried about explanations that postulate unobservables, of which the theory that much thinking is unconscious inference is a paradigm. So psychology suffers from recurring bouts of behaviourism and associationism, to both of which the thinking-as-inferring story is anathema. (p. 16)

According to Fodor, behaviorism and associationism both deny the existence of unobservable events. What nonsense! This feat of theoretical levita- tion may be true of Skinner's early radical behaviorism; it is certainly not true of associationism (remember Hull's "little $r_{g} s$ "?) or theoretical behaviorism. So not only is behaviorism condemned, it is presented as a parody.

I must confess that as I read this piece by Fodor, my first reaction was amazement that something almost selfevidently nonsensical —or at least going wildly beyond the scientific evidence-could be published at all, let alone published in a respected journal with a readership much wider than The Behavior Analyst. But my conclusion after reflection was much worse. If this sort of stuff can be accepted as selfevidently true, behaviorism really is in trouble. We are in trouble not because behaviorism is wrong, but because we have drawn our wagons into a circle and now speak - and shoot-mainly at one another rather than at the wide world outside. Self-criticism is great, but critical misrepresentation by nonbehaviorists requires more. It is time to take articles like Fodor's seriously-to respond to them, rather than ignoring them in the vain hope that truth unaided will prevail.

\section{REFERENCES}

Fodor, J. (2003, October 9). The lemma dilemma. Review of Thinking Without Words by José Luis Bermúdez. London Review of Books, 25 (19), 16-17.

Simon, H. A. (1957). Models of man. New York: Wiley. 